\title{
Hopf bifurcation analysis and its preliminary control in a Hasting-Powell food chain model with two different delays
}

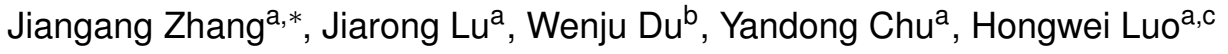 \\ a School of Mathematics and Physics, Lanzhou Jiaotong University, Lanzhou 730070, China. \\ ${ }^{b}$ School of Traffic and Transportation, Lanzhou Jiaotong University, Lanzhou 730070, China. \\ ${ }^{c}$ Department of Information Engineering, Gansu Forestry Technological College, Tianshui, Gansu 741020, China. \\ Communicated by Y. Z. Chen
}

\begin{abstract}
Keeping the balance of nature is important, and it is very significant to effectively control the number of species for ecosystem stability. In this paper, we propose a tritrophic Hastings-Powell (HP) model with two different time delays, and the local stability of equilibrium, Hopf bifurcation, and the existence and uniqueness of the positive equilibrium are analyzed in detail. Besides, we obtain the stable conditions for the system and prove that Hopf bifurcation will occur when the delay pass through the critical value. And the stability and direction of the Hopf bifurcation are also investigated by using the center manifold theorem and normal form theorem. Finally, some numerical examples are given to illustrate the results. (C)2017 All rights reserved.
\end{abstract}

Keywords: Tritrophic Hastings-Powell model, local stability, delays, Hopf bifurcation.

2010 MSC: 74H55, 74H60.

\section{Introduction}

Biological system is a nonlinear system, so it is still a public problem how to control the biological system balance. The predecessors have done a lot of research. Especially, the research on the predatorprey system's dynamic behaviors has attracted significant attention from scholars. And there are also a large number of researches on the stability of predator-prey systems with time delays. The time delays have very complex impact on the dynamic behaviors of nonlinear dynamic systems [2, 3, 11, 15, 17-19]. Zhang [16] produced a general example of such generalized model, that is to say, he investigated a threespecies model, and the results showed that the positive equilibrium is locally stable when the system has two same time delays.

Hastings and Powell [9] proposed a three species food-chain model with chaotic dynamical behavior, in 1991, and then the dynamic properties of the model were studied. Gakkhar and Singh [6] studied

\footnotetext{
${ }^{*}$ Corresponding author

Email addresses: zhangjg7715776@126.com (Jiangang Zhang), ljrong1203@126.com (Jiarong Lu), duwenjuok@126.com (Wenju $\mathrm{Du})$, cyd@mail.lzjtu.cn (Yandong Chu), lhw1220@126.com (Hongwei Luo)

doi:10.22436/jnsa.010.08.14
} 
the control of the chaos of a three species Hastings-Powell food-chain model. The stability of biological feasible equilibrium points of the modified food-web model is also investigated. By introducing the disease in prey population, Das et al. [5] modified the Hastings-Powell model and obtained the stability of biological feasible equilibria. Tian et al. [13] mainly focused on the direction and stability of time-delayinduced Hopf bifurcation in a three species food-chain model with diffusion. Analysis of eigenvalue spectrum and Lyapunov functional of their results show that the positive equilibrium is asymptotically stable in the absence of time delay, but loses its stability via the Hopf bifurcation when the time delay increases beyond a threshold. Chen et al. [4] studied the conditions of the positive equilibrium undergoing Hopf bifurcation by the theory of delay equations and Hopf bifurcation. The stability and direction of Hopf bifurcation are determined by applying normal form theory and the center manifold argument.

In this paper, we provide a differential model [4] to describe the dynamics of the Hastings-Powell food-chain model. In the three species food-chain model, $x$ represents the prey, and $y, z$ represent two predators. Based on the Holling type II functional response, we know that the middle predator $y$ feeds on the prey $x$ and the top predator $z$ preys upon $y$. We can write the three species food chain model as follows [12]:

$$
\begin{aligned}
& \frac{d X}{d T}=R_{0} X\left(1-\frac{X}{K_{0}}\right)-C_{1} \frac{A_{1} X Y}{B_{1}+X} \\
& \frac{d Y}{d T}=-D_{1} Y+\frac{A_{1} X Y}{B_{1}+X}-\frac{A_{2} Y Z}{B_{2}+Y} \\
& \frac{d Z}{d T}=-D_{2} Z+C_{2} \frac{A_{2} Y Z}{B_{2}+Y}
\end{aligned}
$$

where $X, Y, Z$ are the prey, predator and top-predator, respectively; $B_{1}, B_{2}$ represent the half-saturation constants; $R_{0}, K_{0}$ represent the intrinsic growth rate and the carrying capacity of the environment of the prey species, respectively; $C_{1}, C_{2}$ are the conversion factors of prey-to-predator; and $D_{1}, D_{2}$ represent the death rates of $Y$ and $Z$, respectively. In this paper, two different delays are incorporated into the tritrophic Hastings-Powell (HP) model, which is given in what follows.

Then, we introduce the following dimensionless version of the delayed HP model

$$
\begin{aligned}
& \frac{d x}{d t}=x(1-x)-\frac{a_{1} x}{1+b_{1} x} y\left(t-\tau_{1}\right), \\
& \frac{d y}{d t}=-d_{1} y+\frac{a_{1} x}{1+b_{1} x} y-\frac{a_{2} x}{1+b_{2} x} z\left(t-\tau_{2}\right), \\
& \frac{d z}{d t}=-d_{2} z+\frac{a_{2} x}{1+b_{2} x} z,
\end{aligned}
$$

where $x, y$ and $z$ represent dimensionless population variables; $t$ represents dimensionless time variable and all of the parameters $a_{i}, b_{i}, d_{i}(i=1,2)$ are positive; $\tau_{1}$ and $\tau_{2}$ respectively represent the period of prey transitioned to predator and predator transitioned to the top predator.

\section{Equilibrium and local stability analysis}

Let $\dot{x}=0, \dot{y}=0, \dot{z}=0$; we can get five non-negative equilibrium points of the system as follows

$$
\begin{gathered}
E_{0}=(0,0,0), \quad E_{1}=(1,0,0), \\
E_{2}=\left(\frac{d_{1}}{a_{1}-b_{1} d_{1}}, \frac{a_{1}-b_{1} d_{1}-d_{1}}{\left(a_{1}-b_{1} d_{1}\right)^{2}}, 0\right), \quad E_{3,4}=\left(\bar{x}_{i}, \bar{y}_{i}, \bar{z}_{i}\right), \quad i=1,2,
\end{gathered}
$$

where

$$
\begin{aligned}
& \bar{x}_{i}=\frac{b_{1}-1}{2 b_{1}}+(-1)^{i-1} \frac{\sqrt{\left(b_{1}+1\right)^{2}-\frac{4 a_{1} b_{1} d_{2}}{a_{2}-b_{2} d_{2}}}}{2 b_{1}}, \\
& y_{1}=\bar{y}_{2}=\frac{d_{2}}{a_{2}-b_{2} d_{2}}, \quad \bar{z}_{i}=\frac{\left(a_{1}-b_{1} d_{1}\right) \bar{x}_{i}-d_{1}}{\left(a_{2}-b_{2} d_{2}\right)\left(1+b_{1} \bar{x}_{i}\right)} .
\end{aligned}
$$


The Jacobian matrix for system (1.1) at $E^{*}=\left(x^{*}, y^{*}, z^{*}\right)$ is

$$
J\left(x^{*}, y^{*}, z^{*}\right)=\left(\begin{array}{lll}
1-2 x-\frac{a_{1} y}{\left(1+b_{1} x\right)^{2}} & -\frac{a_{1} x}{1+b_{1} x} & 0 \\
\frac{a_{1} y}{\left(1+b_{1} x\right)^{2}} & -d_{1}+\frac{a_{1} x}{1+b_{1} x}-\frac{a_{1} z}{\left(1+b_{1} y\right)^{2}} & -\frac{a_{2} y}{1+b_{2} y} \\
0 & \frac{a_{1} z}{\left(1+b_{1} y\right)^{2}} & -d_{2}+\frac{a_{2} y}{1+b_{2} y}
\end{array}\right) .
$$

Let

$$
\begin{aligned}
& A_{1}=1-2 x-\frac{a_{1} y}{\left(1+b_{1} x\right)^{2}}, \quad A_{2}=-\frac{a_{1} x}{1+b_{1} x}, \\
& B_{1}=\frac{a_{1} y}{\left(1+b_{1} x\right)^{2}}, \quad B_{2}=-d_{1}+\frac{a_{1} x}{1+b_{1} x}-\frac{a_{1} z}{\left(1+b_{1} y\right)^{2}}, \\
& B_{3}=-\frac{a_{2} y}{1+b_{2} y}, \quad C_{2}=\frac{a_{2} z}{\left(1+b_{2} y\right)^{2}}, \quad C_{3}=-d_{2}+\frac{a_{2} y}{1+b_{2} y} .
\end{aligned}
$$

Then the linearized form of system (1.2) is

$$
\begin{aligned}
& \frac{d x}{d t}=A_{1} x+A_{2} y\left(t-\tau_{1}\right), \\
& \frac{d y}{d t}=B_{1} x+B_{2} y+B_{3} z\left(t-\tau_{2}\right), \\
& \frac{d z}{d t}=C_{2} y+C_{3} z .
\end{aligned}
$$

The characteristic equation of system $(2.4)$ at $E_{0}=(0,0,0)$ is given by the transcendental equation

$$
\lambda^{3}+A_{11} \lambda^{2}+A_{12} \lambda+A_{13}+\left(A_{21} \lambda+A_{22}\right) e^{-\lambda \tau_{1}}+\left(A_{31} \lambda+A_{32}\right) e^{-\lambda \tau_{2}}=0,
$$

where

$$
\begin{aligned}
& A_{11}=-\left(A_{1}+B_{2}+C_{3}\right), \quad A_{12}=A_{1} B_{2}+A_{1} C_{3}+B_{2} C_{3}, \quad A_{13}=-A_{1} B_{2} C_{3}, \\
& A_{21}=-A_{2} B_{1}, \quad A_{22}=A_{2} B_{1} C_{3}, \quad A_{31}=-B_{3} C_{2}, \quad A_{32}=A_{1} B_{3} C_{2} .
\end{aligned}
$$

When $\tau_{1}=\tau_{2}=0$, the corresponding characteristic (2.5) can be rewritten as

$$
\lambda^{3}+A_{11} \lambda^{2}+\left(A_{12}+A_{21}+A_{31}\right) \lambda+A_{13}+A_{22}+A_{32}=0 .
$$

Lemma 2.1. Suppose that the following conditions hold

1. $A_{11}>0$;

2. $A_{11}\left(A_{12}+A_{21}+A_{31}\right)>A_{13}+A_{22}+A_{32}$.

Then the positive equilibrium $\mathrm{E}^{*}$ of system (1.2) is locally asymptotically stable for $\tau_{1}$ and $\tau_{2}$.

\section{Existence of Hopf bifurcation}

Case I: $\tau_{1}=\tau_{2}=\tau \neq 0$.

Characteristic (2.5) reduces to

$$
\lambda^{3}+A_{11} \lambda^{2}+A_{12} \lambda+A_{13}+\left(B_{11} \lambda+B_{12}\right) e^{-\lambda \tau}=0,
$$

where

$$
B_{11}=A_{21}+A_{31}, \quad B_{12}=A_{22}+A_{32} .
$$

Let $\lambda=i \omega(\omega>0)$ be a root of (3.1). Then we can get

$$
(i \omega)^{3}+A_{11}(i \omega)^{2}+A_{12} i \omega+A_{13}+\left(B_{11} i \omega+B_{12}\right) e^{-i \omega \tau}=0 .
$$


Separating the real and imaginary parts, we have

$$
\left\{\begin{array}{l}
B_{12} \cos \omega \tau-B_{11} \omega \sin \omega \tau=A_{11} \omega^{2}-A_{13} \\
B_{11} \omega \cos \omega \tau+B_{12} \sin \omega \tau=\omega^{3}-A_{12} \omega .
\end{array}\right.
$$

From (3.2), we can get

$$
\begin{aligned}
& \sin \omega \tau=-\frac{\left(A_{11} B_{11}-B_{12}\right) \omega^{3}+\left(A_{12} B_{12}-A_{13} B_{11}\right) \omega}{B_{11}^{2} \omega^{2}+B_{12}^{2}}, \\
& \cos \omega \tau=\frac{B_{11} \omega^{4}+\left(B_{12} A_{11}-A_{12} B_{11}\right) \omega^{2}-A_{13} B_{12}}{B_{11}^{2} \omega^{2}+B_{12}^{2}} .
\end{aligned}
$$

That is,

$$
a w^{8}+b w^{6}+c \omega^{4}+d w^{2}+k=0
$$

where

$$
\begin{aligned}
a= & B_{11}^{2}, \\
b= & \left(A_{11} B_{11}-B_{12}\right)^{2}+2\left(A_{11} B_{12}-A_{12} B_{11}\right) \\
c= & -B_{11}^{2}+2\left(A_{12} B_{12}-A_{13} B_{11}\right)\left(A_{11} B_{11}-B_{12}\right)-2 A_{13} B_{11} B_{12} \\
& +\left(A_{11} B_{12}-A_{12} B_{11}\right)^{2} \\
k= & B_{12}^{2} A_{13}^{2}-B_{12}^{4} \\
d= & 2 B_{11}^{2} B_{12}^{2}+\left(A_{12} B_{12}-A_{13} B_{11}\right)^{2}-2 A_{13} B_{12}\left(A_{11} B_{12}-A_{12} B_{11}\right),
\end{aligned}
$$

Let $z=\omega^{2}$; then we have

$$
\mathrm{a} z^{4}+\mathrm{b} z^{3}+\mathrm{c} z^{2}+\mathrm{d} z+\mathrm{k}=0
$$

Now suppose that $H(z)=a z^{4}+b z^{3}+c z^{2}+d z+k$. Then we have the following result.

Lemma 3.1. Since $\mathrm{H}(+\infty)=+\infty$, if the condition $\mathrm{H}(0)<0$ holds, then (3.5) has at least one positive root, and we suppose that (3.5) has four positive roots denoted by $z_{1}, z_{2}, z_{3}, z_{4}$. Therefore (3.4) also has four positive roots $\omega_{\mathrm{k}}=\sqrt{z_{\mathrm{k}}}, \mathrm{k}=1,2,3,4$.

Then $\pm i \omega$ is a pair of purely imaginary roots of (3.1). Solving (3.3) for $\tau$, we obtain

$$
\tau_{k}^{(j)}=\frac{1}{\omega_{k}}\left[\arccos \left(\frac{B_{11} \omega^{4}+\left(B_{12} A_{11}-A_{12} B_{11}\right) \omega^{2}-A_{13} B_{12}}{B_{11}^{2} \omega^{2}+B_{12}^{2}}\right)+2 j \pi\right], \quad k=1,2,3,4, j=0,1,2, \cdots .
$$

Define $\tau_{0}=\tau_{k}^{(j)}=\min _{k \in\{1,2,3,4\}}\left\{\tau_{k}^{(0)}\right\}$. Let $\lambda(\tau)=\alpha(\tau)+i \omega(\tau)$ be the root of (3.1) near $\tau=\tau_{k}$, satisfying $\alpha\left(\tau_{k}\right)=0$ and $\omega\left(\tau_{k}\right)=\omega_{0}$, and from above, we can obtain the following lemma.

Lemma 3.2. Suppose that $\mathrm{H}^{\prime}(z) \neq 0$; then

$$
\left.\left[\frac{\mathrm{d} \operatorname{Re} \lambda(\tau)}{\mathrm{d} \tau}\right]\right|_{\tau=\tau_{k}} \neq 0
$$

and $\mathrm{H}^{\prime}(z)$ and $\frac{\mathrm{d} \operatorname{Re} \lambda(\tau)}{\mathrm{d} \tau}$ have the same sign.

Proof. Taking the derivative of $\lambda$ with respect to $\tau$ in (3.1), we have

$$
\left[\frac{d \lambda}{d \tau}\right]^{-1}=\frac{\left(3 \lambda^{2}+2 A_{11} \lambda+A_{12}\right) e^{\lambda \tau}}{\left(B_{11} \lambda+B_{12}\right) \lambda}+\frac{B_{11}}{\left(B_{11} \lambda+B_{12}\right) \lambda}-\frac{\tau}{\lambda} .
$$

Substituting $\lambda(\tau)=\alpha(\tau)+i \omega(\tau)$ into (3.7), we have

$$
\begin{aligned}
{\left.\left[\left(3 \lambda^{2}+2 A_{11} \lambda+A_{12}\right) e^{\lambda \tau}\right]\right|_{\lambda}=} & i \omega_{k}=\left(A_{12}-3 \omega^{2}\right) \cos \omega \tau-2 A_{11} \omega \sin \omega \tau \\
& +i\left[\left(A_{12}-3 \omega^{2}\right) \sin \omega \tau-2 A_{11} \omega \cos \omega \tau\right],
\end{aligned}
$$




$$
\left.\left[\left(B_{11} \lambda+B_{12}\right) \lambda\right]\right|_{\lambda=i \omega_{k}}=-B_{11} \omega^{2}+i\left[B_{12} \omega\right] .
$$

For simplicity, we define $\omega_{k}=\omega$ and $\tau_{k}=\tau$. From (3.7), (3.8), (3.9) and (3.3), we can get

$$
\begin{aligned}
{\left[\frac{d \operatorname{Re} \lambda(\tau)}{d \tau}\right]^{-1}=} & {\left.\left[\frac{\left(3 \lambda^{2}+2 A_{11} \lambda+A_{12}\right) e^{\lambda \tau}+B_{11}}{\left(B_{11} \lambda+B_{12}\right) \lambda}\right]\right|_{\lambda=i \omega} } \\
= & \operatorname{Re}\left\{\frac{\left(A_{12}-3 \omega^{2}\right) \cos \omega \tau-2 A_{11} \omega \sin \omega \tau+B_{11}+i\left[\left(A_{12}-3 \omega^{2}\right) \sin \omega \tau-2 A_{11} \omega \cos \omega \tau\right]}{-B_{11} \omega^{2}+i\left[B_{12} \omega\right]}\right\} \\
= & \frac{1}{\Delta}\left\{\left[\left(A_{12}-3 \omega^{2}\right) \cos \omega \tau-2 A_{11} \omega \sin \omega \tau+B_{11}\right]\left(-B_{11} \omega^{2}\right)\right. \\
& \left.+\left[\left(A_{12}-3 \omega^{2}\right) \sin \omega \tau-2 A_{11} \omega \cos \omega \tau\right] B_{12} \omega\right\} \\
= & \frac{1}{\Delta}\left\{4 B_{11}{ }^{2} \omega^{8}+3\left[\left(A_{11} B_{11}-B_{12}\right)^{2}+2\left(A_{11} B_{12}-A_{12} B_{11}\right)\right] \omega^{6}\right. \\
& +2\left[2\left(A_{12} B_{12}-A_{13} B_{11}\right)\left(A_{11} B_{11}-B_{12}\right)-B_{11}{ }^{2}-2 A_{13} B_{11} B_{12}\right] \omega^{4} \\
& +2\left[\left(A_{11} B_{12}-A_{12} B_{11}\right)^{2}\right] \omega^{4}+\left[\left(A_{12} B_{12}-A_{13} B_{11}\right)^{2}+2 B_{11}{ }^{2} B_{12}{ }^{2}\right] \omega^{2} \\
& \left.-2 A_{13} B_{12}\left(A_{11} B_{12}-A_{12} B_{11}\right) \omega^{2}\right\} \\
= & \frac{z}{\Delta}\left\{4 B_{11}^{2} z^{3}+3\left[\left(A_{11} B_{11}-B_{12}\right)^{2}+2\left(A_{11} B_{12}-A_{12} B_{11}\right)\right] z^{2}\right. \\
& +2\left[2\left(A_{12} B_{12}-A_{13} B_{11}\right)\left(A_{11} B_{11}-B_{12}\right)-B_{11}{ }^{2}-2 A_{13} B_{11} B_{12}\right] z \\
& +\left(A_{11} B_{12}-A_{12} B_{11}\right)^{2} z+\left(A_{12} B_{12}-A_{13} B_{11}\right)^{2}+2 B_{11}{ }^{2} B_{12}{ }^{2} \\
& \left.-2 A_{13} B_{12}\left(A_{11} B_{12}-A_{12} B_{11}\right)\right\} \\
= & \frac{z}{\Delta} H^{\prime}(z) \\
&
\end{aligned}
$$

where $\Delta=\mathrm{B}_{11}^{2} \omega^{4}+\mathrm{B}_{12}^{2} \omega^{2}$. Then, we have,

$$
\left.\operatorname{sign}\left[\frac{\mathrm{d} \operatorname{Re} \lambda(\tau)}{\mathrm{d} \tau}\right]\right|_{\tau=\tau_{k}}=\left.\operatorname{sign}\left[\frac{\mathrm{d} \operatorname{Re} \lambda(\tau)}{\mathrm{d} \tau}\right]^{-1}\right|_{\tau=\tau_{k}}=\operatorname{sign}\left[\frac{z}{\Delta} \mathrm{H}^{\prime}(z)\right] \neq 0 .
$$

This proves the lemma.

By applying Lemmas 3.1-3.2, we get the following theorem.

Theorem 3.3. For system (1.2), the following results hold:

(i) At equilibrium point $\mathrm{E}^{*}=\left(\mathrm{x}^{*}, \mathrm{y}^{*}, \mathrm{z}^{*}\right)$, system (1.2) is asymptotically stable for $\tau \in\left[0, \tau_{0}\right)$, and it is unstable when $\tau>\tau_{0}$.

(ii) If system (1.2) meets the conditions of Lemmas 3.1 and 3.2 , then the Hopf bifurcation will occur at $\mathrm{E}^{*}\left(\mathrm{x}^{*}, \mathrm{y}^{*}, z^{*}\right)$ when $\tau=\tau_{0}$.

Case II: $\tau_{1} \neq 0, \tau_{2}=0$.

Let $D_{11}=A_{12}+A_{31}, C_{11}=A_{13}+A_{32}$, and rewrite (2.4) as follows

$$
\lambda^{3}+A_{11} \lambda^{2}+D_{11} \lambda+C_{11}+\left(A_{21} \lambda+A_{22}\right) e^{-\lambda \tau_{1}}=0 .
$$


Let $\lambda=i \omega(\omega>0)$ be a root of (3.10); we can get

$$
\left\{\begin{array}{l}
A_{22} \cos \omega \tau_{1}-A_{21} \omega \sin \omega \tau_{1}=A_{11} \omega^{2}-C_{11} \\
A_{21} \omega \cos \omega \tau_{1}+A_{22} \sin \omega \tau_{1}=\omega^{3}-D_{11} \omega
\end{array}\right.
$$

Similarly, we have

$$
a_{1} \omega^{8}+b_{1} \omega^{6}+c_{1} \omega^{4}+d_{1} \omega^{2}+k_{1}=0,
$$

where

$$
\begin{aligned}
a_{1}= & A_{21}^{2}, \\
b_{1}= & \left(A_{11} A_{21}-A_{22}\right)^{2}+2\left(A_{11} A_{22}-D_{11} A_{21}\right) \\
c_{1}= & -A_{21}^{2}+2\left(D_{11} A_{22}-C_{11} A_{21}\right)\left(A_{11} A_{21}-A_{22}\right)-2 C_{11} A_{21} A_{22} \\
& +\left(A_{11} A_{22}-D_{11} A_{21}\right)^{2} \\
k_{1}= & A_{22}^{2} C_{11}^{2}-A_{22}^{4} \\
d_{1}= & 2 A_{21}^{2} A_{22}^{2}+\left(D_{11} A_{22}-C_{11} A_{21}\right)^{2}-2 C_{11} A_{22}\left(A_{11} A_{22}-D_{11} A_{21}\right) .
\end{aligned}
$$

If we define $z_{1}=\omega^{2}$, then equation (3.12) becomes

$$
a_{1} z_{1}^{4}+b_{1} z_{1}^{3}+c_{1} z_{1}^{2}+d_{1} z_{1}+k_{1}=0 .
$$

Now suppose that $H\left(z_{1}\right)=a_{1} z_{1}^{8}+b_{1} z_{1}^{6}+c_{1} z_{1}^{4}+d_{1} z_{1}^{2}+k_{1}$. The we have the following.

Lemma 3.4. It is obvious that $\mathrm{H}(+\infty)=+\infty$. If the condition $\mathrm{H}(0)<0$ holds, then (3.5) has at least one positive root. Suppose that (3.5) has four positive roots denoted by $z_{11}, z_{12}, z_{13}, z_{14}$. Then (3.4) also has four positive roots $\omega_{k}=\sqrt{z_{1 k}}, k=1,2,3,4$.

Then $\pm i \omega$ is a pair of purely imaginary roots of (3.1). From (3.11), if we denote

$$
\tau_{1 k}^{(j)}=\frac{1}{\omega_{k}}\left[\arccos \left(\frac{A_{21} \omega^{4}+\left(A_{22} A_{11}-D_{11} A_{21}\right) \omega^{2}-C_{11} A_{22}}{A_{21}^{2} \omega^{2}+A_{22}^{2}}\right)+2 j \pi\right], \quad k=1,2,3,4, j=0,1,2, \cdots,
$$

then we can define $\tau_{10}=\tau_{1 \mathrm{k}}^{(\mathrm{j})}=\min _{\mathrm{k} \in\{1,2,3,4\}}\left\{\tau_{1 \mathrm{k}}^{(0)}\right\}$. Also denote

$$
\begin{aligned}
\mathrm{P}= & {\left[\left(3 \lambda^{2}+2 A_{11} \lambda+D_{11}\right) e^{\lambda \tau_{1}}\right]_{\lambda=i \omega_{k}} } \\
= & \left(D_{11}-3 \omega^{2}\right) \cos \omega \tau_{1}-2 A_{11} \omega \sin \omega \tau_{1} \\
& +i\left[\left(D_{11}-3 \omega^{2}\right) \sin \omega \tau_{1}-2 A_{11} \omega \cos \omega \tau_{1}\right] \\
:= & P_{R}+i P_{I}, \\
Q= & {\left[\left(A_{21} \lambda+A_{22}\right)\right]=-A_{21} \omega^{2}+i A_{22} \omega:=Q_{R}+i Q_{I} . }
\end{aligned}
$$

Let $\lambda(\tau)=\alpha(\tau)+i \omega(\tau)$ be a root of (3.1) near $\tau=\tau_{10}$, satisfying $\alpha\left(\tau_{10}\right)=0$ and $\omega\left(\tau_{10}\right)=\omega_{0}$.

From above, we can obtain the following lemma.

Lemma 3.5. Suppose that $\mathrm{P}_{\mathrm{R}} \mathrm{Q}_{\mathrm{R}}+\mathrm{P}_{\mathrm{I}} \mathrm{Q}_{\mathrm{I}} \neq 0$ holds; then

$$
\left.\left[\frac{\mathrm{d} \operatorname{Re} \lambda\left(\tau_{10}\right)}{\mathrm{d} \tau_{1}}\right]\right|_{\tau=\tau_{1 \mathrm{k}}} \neq 0 .
$$

Proof. Taking the derivative of $\lambda$ with respect to $\tau_{1}$ in (3.9), we can get

$$
\left[\frac{d \lambda}{d \tau_{1}}\right]^{-1}=\operatorname{Re}\left[\frac{\left(3 \lambda^{2}+2 A_{11} \lambda+A_{12}\right) e^{\lambda \tau_{1}}}{\left(A_{21} \lambda+A_{22}\right) \lambda}+\frac{A_{21}}{\left(A_{21} \lambda+A_{22}\right) \lambda}-\frac{\tau_{1}}{\lambda}\right] .
$$


By substituting $\lambda=i \omega$ into (3.14) we have

$$
\begin{aligned}
{\left[\frac{\mathrm{d} \operatorname{Re} \lambda}{\mathrm{d} \tau_{1}}\right]_{\tau=\tau_{1 k}}^{-1} } & =\operatorname{Re}\left[\frac{\left(3 \lambda^{2}+2 A_{11} \lambda+A_{12}\right) e^{\lambda \tau_{1}}}{\left(A_{21} \lambda+A_{22}\right) \lambda}+\frac{A_{21}}{\left(A_{21} \lambda+A_{22}\right) \lambda}-\frac{\tau_{1}}{\lambda}\right]_{\tau=\tau_{1 k}} \\
& =\frac{P_{R} Q_{R}+P_{I} Q_{I}}{P_{R}^{2}+P_{I}^{2}} .
\end{aligned}
$$

Since $P_{R} Q_{R}+P_{I} Q_{I} \neq 0$ holds, we obtain

$$
\left.\left[\frac{\mathrm{d} \operatorname{Re} \lambda\left(\tau_{10}\right)}{\mathrm{d} \tau_{1}}\right]\right|_{\tau=\tau_{1 k}} \neq 0
$$

This proves the lemma.

By applying Lemmas 3.4 and 3.5, we can prove the existence of Hopf bifurcation.

Theorem 3.6. For system (1.2), the following results hold:

(i) At the equilibrium point $\mathrm{E}^{*}\left(\mathrm{x}^{*}, \mathrm{y}^{*}, z^{*}\right)$, system (1.2) is asymptotically stable for $\tau_{1} \in\left[0, \tau_{10}\right)$, and it is unstable for $\tau_{1}>\tau_{10}$.

(ii) If system (1.2) satisfies the conditions of Lemmas 3.4 and 3.5, then system (1.2) undergoes the Hopf bifurcation at $\mathrm{E}^{*}\left(\mathrm{x}^{*}, \mathrm{y}^{*}, z^{*}\right)$ when $\tau_{1}=\tau_{10}$.

Case III: $\tau_{1}=0, \tau_{2} \neq 0$.

Equation (2.5) can be written as

$$
\lambda^{3}+A_{11} \lambda^{2}+D_{12} \lambda+C_{12}+\left(A_{31} \lambda+A_{32}\right) e^{-\lambda \tau_{2}}=0
$$

where $D_{12}=A_{12}+A_{21}, C_{12}=A_{13}+A_{22}$.

Let $\lambda=i \omega(\omega>0)$ be a root of (3.16); we can get

$$
\left\{\begin{array}{l}
A_{32} \cos \omega \tau_{2}-A_{31} \omega \sin \omega \tau_{2}=A_{11} \omega^{2}-C_{12} \\
A_{31} \omega \cos \omega \tau_{2}+A_{32} \sin \omega \tau_{2}=\omega^{3}-D_{12} \omega
\end{array}\right.
$$

Then we can get

$$
a_{2} \omega^{8}+b_{2} \omega^{6}+c_{2} \omega^{4}+d_{2} \omega^{2}+k_{2}=0,
$$

where

$$
\begin{aligned}
a_{2}= & A_{31}^{2}, \\
b_{2}= & \left(A_{11} A_{31}-A_{32}\right)^{2}+2\left(A_{11} A_{32}-D_{12} A_{31}\right), \\
c_{2}= & -A_{31}^{2}+2\left(D_{12} A_{32}-C_{12} A_{31}\right)\left(A_{11} A_{31}-A_{32}\right)-2 C_{12} A_{31} A_{32} \\
& +\left(A_{11} A_{32}-D_{12} A_{31}\right)^{2} \\
k_{2}= & A_{32}^{2} C_{12}^{2}-A_{32}^{4} \\
d_{2}= & 2 A_{31}^{2} A_{32}^{2}+\left(D_{12} A_{32}-C_{12} A_{31}\right)^{2}-2 C_{12} A_{32}\left(A_{11} A_{32}-D_{12} A_{31}\right) .
\end{aligned}
$$

Letting $z_{2}=\omega^{2}$, equation (3.18) becomes

$$
\mathrm{a}_{2} z_{2}^{4}+\mathrm{b}_{2} z_{2}^{3}+\mathrm{c}_{2} z_{2}^{2}+\mathrm{d}_{2} z_{2}+\mathrm{k}_{2}=0 .
$$

Now let $H\left(z_{2}\right)=a_{2} z_{2}^{4}+b_{2} z_{2}^{3}+c_{2} z_{2}^{2}+d_{2} z_{2}+k_{2}$. Then we have the following. 
Lemma 3.7. Since $\mathrm{H}(+\infty)=+\infty$, and if $\mathrm{H}(0)<0$, then (3.19) has at least one positive root. Suppose that (3.19) has four positive roots denoted by $z_{21}, z_{22}, z_{23}, z_{24}$. Then (3.18) also has four positive roots $\omega_{k}=\sqrt{z_{2 k}}, k=$ $1,2,3,4$.

Then $\pm i \omega$ is a pair of purely imaginary roots of (3.16). From (3.17), if we denote

$$
\tau_{2 k}^{(j)}=\frac{1}{\omega_{k}}\left[\arccos \left(\frac{A_{31} \omega^{4}+\left(A_{32} A_{11}-D_{12} A_{31}\right) \omega^{2}-C_{12} A_{32}}{A_{31}^{2} \omega^{2}+A_{32}^{2}}\right)+2 j \pi\right], \quad k=1,2,3,4, j=0,1,2, \cdots,
$$

then we can define $\tau_{20}=\tau_{2 \mathrm{k}}^{(\mathrm{j})}=\min _{\mathrm{k} \in\{1,2,3,4\}}\left\{\tau_{2 \mathrm{k}}^{(0)}\right\}$.

Let $\lambda(\tau)=\alpha(\tau)+i \omega(\tau)$ be a root of (3.1) near $\tau=\tau_{20}$, satisfying $\alpha\left(\tau_{20}\right)=0$ and $\omega\left(\tau_{20}\right)=\omega_{0}$ From the above analysis, we can obtain the following lemma.

Lemma 3.8. Suppose that $z_{2}=\omega^{2}$; then

$$
\left.\left[\frac{\mathrm{d} \operatorname{Re} \lambda\left(\tau_{2}\right)}{\mathrm{d} \tau_{2}}\right]\right|_{\tau=\tau_{2 k}} \neq 0 .
$$

Proof. This proof is similar to the proof of Lemma 3.5, so we omit it.

According to Lemmas 3.7 and 3.8 to (3.16), we get the following theorem.

Theorem 3.9. For system (1.2), the following results hold:

(i) $E^{*}\left(x^{*}, y^{*}, z^{*}\right)$ is asymptotically stable when $\tau_{2} \in\left[0, \tau_{20}\right)$, and unstable when $\tau_{2}>\tau_{20}$.

(ii) If system (1.2) meets the conditions of Lemmas 3.7 and 3.8, then the Hopf bifurcation occurs at $\mathrm{E}^{*}\left(x^{*}, \mathrm{y}^{*}, z^{*}\right)$ when $\tau_{2}=\tau_{20}$.

Case IV: $\tau_{1} \neq \tau_{2} \neq 0$.

We consider (2.5) with $\tau_{1}$ in the stability range. Regarding $\tau_{2}$ as a parameter, and without loss of generality, we only consider system (1.2) under Case I.

Let $\lambda=i \omega(\omega>0)$ be a root of (2.5); we have

$$
\left\{\begin{array}{l}
A_{32} \cos \omega \tau_{2}+A_{31} \omega \sin \omega \tau_{2}=A_{11} \omega^{2}-A_{13}-\left(A_{22} \cos \omega \tau_{1}+A_{12} \omega \sin \omega \tau_{1}\right), \\
A_{31} \omega \cos \omega \tau_{2}+A_{32} \sin \omega \tau_{2}=\omega^{3}-A_{12} \omega-\left(A_{12} \omega \cos \omega \tau_{1}-A_{22} \sin \omega \tau_{1}\right) .
\end{array}\right.
$$

Eliminating $\tau_{2}$ from (3.21) leads to

$$
y_{1}(\omega)+y_{2}(\omega) \cos \omega \tau_{1}+y_{3}(\omega) \sin \omega \tau_{1}=0
$$

Lemma 3.10. Suppose that (3.22) has positive roots. Denote the roots of (3.22) by $z_{31}, z_{32}, \ldots, z_{3 k}$. Then (3.18) also has positive roots $\omega_{k}=\sqrt{z_{3 i}}, i=1,2, \cdots, k$. If we denote

$$
\tau_{3 i}^{(j)}=\frac{1}{\omega_{i}}\left[\arccos \left(\frac{\psi_{1}}{\psi_{2}}\right)+2 j \pi\right], \quad i=1,2, \cdots k, j=0,1,2, \cdots,
$$

where

$$
\begin{aligned}
\psi_{1}= & A_{31} \omega^{4}+\left(A_{32} A_{11}-A_{31} A_{12}\right) \omega^{2}-\left(A_{22} A_{32}+A_{31} A_{12} \omega^{2}\right) \cos \omega \tau_{1} \\
& +\left(A_{31} A_{22}-A_{32} A_{12}\right) \omega \sin \omega \tau_{1} \psi_{2} \\
= & A_{31} \omega^{2}+A_{32}^{2}
\end{aligned}
$$

then $\pm i \omega$ is a pair of purely imaginary roots of (2.5). 
Define $\tau_{30}=\tau_{3 i}^{(j)}=\min \left\{\tau_{3 i}^{(j)} \mid i=1,2, \cdots k, j=0,1,2, \cdots\right\}$. Let $\lambda(\tau)=\alpha(\tau)+i \omega(\tau)$ be a root of (3.1) near $\tau=\tau_{30}$, satisfying $\alpha\left(\tau_{30}\right)=0$ and $\omega\left(\tau_{30}\right)=\omega_{0}$. From the above analysis, we can get the following lemma, which is similar to Lemma 3.5 if we denote

$$
\begin{aligned}
& Q_{R}=-3 \omega^{2}+A_{12}+\left(A_{21}-A_{22} \tau_{1}\right) \cos \omega \tau_{1}-A_{21} \omega \tau_{1} \sin \omega \tau_{1} \\
&+\left(A_{31}-A_{32} \tau_{2}\right) \cos \omega \tau_{2}-A_{31} \omega \tau_{2} \sin \omega \tau_{2}, \\
& Q_{I}= 2 A_{11} \omega+\left(A_{22} \tau_{1}-A_{21}\right) \sin \omega \tau_{1}-A_{21} \omega \tau_{1} \cos \omega \tau_{1} \\
&+\left(A_{32} \tau_{2}-A_{31}\right) \sin \omega \tau_{2}-A_{31} \omega \tau_{2} \cos \omega \tau_{2}, \\
& P_{R}=-A_{31} \omega^{2} \cos \omega \tau_{2}+A_{32} \omega \sin \omega \tau_{2}, \quad P_{I}=A_{31} \omega^{2} \sin \omega \tau_{2}+A_{32} \omega \cos \omega \tau_{2} .
\end{aligned}
$$

Lemma 3.11. Suppose that $P_{R} Q_{R}+P_{I} Q_{I} \neq 0$ holds; then

$$
\left.\left[\frac{\mathrm{d} \operatorname{Re} \lambda\left(\tau_{2}\right)}{\mathrm{d} \tau_{2}}\right]\right|_{\tau=\tau_{3 i}} \neq 0
$$

By applying Lemmas 3.7 and 3.8 to (3.16) and based on the Hopf bifurcation theorem for FDEs [7, 14], we can get the following theorem.

Theorem 3.12. If system (1.2) satisfies the conditions of Lemmas 3.7 and 3.8 and $\tau_{1} \in\left[0, \tau_{10}\right)$, then the following results hold:

(i) $\mathrm{E}^{*}\left(\mathrm{x}^{*}, \mathrm{y}^{*}, z^{*}\right)$ is asymptotically stable for $\tau_{2} \in\left[0, \tau_{30}\right)$, and unstable when $\tau_{2}>\tau_{30}$.

(ii) If Lemmas 3.10 and 3.11 hold, then the Hopf bifurcation occurs at $\mathrm{E}^{*}\left(x^{*}, \mathrm{y}^{*}, z^{*}\right)$ when $\tau_{2}=\tau_{30}$.

\section{Bifurcation analysis}

We studied that the Hastings-Powell food chain system with delay undergoes the Hopf bifurcation when $\tau=\tau_{0}^{0}$ in the previous section. Next, we will confirm the Hopf bifurcation's stability, direction and the period of the periodic solution bifurcation.

Firstly, we consider system (1.2) by the transformation

$$
\dot{\mathrm{u}}_{1}(\mathrm{t})=x(\mathrm{t})-\mathrm{x}^{*}, \quad \dot{\mathrm{u}}_{2}(\mathrm{t})=\mathrm{y}(\mathrm{t})-\mathrm{y}^{*}, \quad \dot{\mathrm{u}}_{3}(\mathrm{t})=z(\mathrm{t})-z^{*}, \quad \mathrm{t}=\frac{\mathrm{t}}{\tau^{\prime}} \quad \tau=\tau_{k}+\mu .
$$

We get the following system of functional differential equation in $C=C\left([-1,0], R^{3}\right)$ :

$$
\dot{u}(t)=L_{\mu}\left(u_{t}\right)+f\left(\mu, u_{t}\right),
$$

where $u(t)=\left(u_{1}(t), u_{2}(t), u_{3}(t)\right)^{\top} \in R^{3}$, and $L_{\mu}: C \rightarrow R^{2}, f: R \times C \rightarrow R^{3}$ are given respectively by

$$
\begin{aligned}
\mathrm{L}_{\mu}\left(x_{\mathrm{t}}\right)= & \left(\tau_{\mathrm{k}}+\mu\right)\left[\begin{array}{c}
\mathrm{A}_{1} 00 \\
\mathrm{~B}_{1} \mathrm{~B}_{2} 0 \\
0 \mathrm{C}_{2} \mathrm{C}_{3}
\end{array}\right]\left[\begin{array}{l}
\phi_{1}(0) \\
\phi_{2}(0) \\
\phi_{3}(0)
\end{array}\right]+\left(\tau_{k}+\mu\right)\left[\begin{array}{c}
0 A_{2} 0 \\
000 \\
000
\end{array}\right]\left[\begin{array}{c}
\phi_{1}(-1) \\
\phi_{2}(0) \\
\phi_{3}(0)
\end{array}\right] \\
& +\left(\tau_{k}+\mu\right)\left[\begin{array}{c}
000 \\
00 \mathrm{~B}_{3} \\
000
\end{array}\right]\left[\begin{array}{c}
\phi_{1}(0) \\
\phi_{2}(-1) \\
\phi_{3}(0)
\end{array}\right],
\end{aligned}
$$

and

$$
f(\mu, \varphi)=\left(\tau_{k}+\mu\right)\left[\begin{array}{l}
f_{11} \\
f_{12} \\
f_{13}
\end{array}\right] \varphi=\left(\varphi_{1}, \varphi_{2}, \varphi_{3}\right) \in \mathrm{C} .
$$


By Riesz representation theorem $[1,10]$, there exists a function $\eta(\theta, \mu)$ of bounded variation for $\theta \in[-\tau, 0]$ such that

$$
\mathrm{L}_{\mu}(\varphi)=\int_{-\tau}^{0} \mathrm{d \eta}(\theta, \mu) \varphi(\theta), \quad \theta \in \mathrm{C} .
$$

Indeed we may take

$$
\begin{aligned}
\eta(0, \mu)= & \left(\tau_{k}+\mu\right)\left[\begin{array}{c}
A_{1} 00 \\
B_{1} B_{2} 0 \\
0 C_{2} C_{3}
\end{array}\right] \delta(\theta)+\left(\tau_{k}+\mu\right)\left[\begin{array}{c}
0 A_{2} 0 \\
000 \\
000
\end{array}\right] \delta(\theta+1) \\
& +\left(\tau_{k}+\mu\right)\left[\begin{array}{c}
000 \\
00 B_{3} \\
000
\end{array}\right] \delta(\theta+1)
\end{aligned}
$$

where $\delta(\theta)$ is the Dirac delta function. For $\varphi(\theta) \in C\left([-1,0], R^{3}\right)$, we define the operator $A(\mu)$ by

$$
A(\mu) \varphi(\theta)=\left\{\begin{array}{lc}
\frac{d}{d \theta}, & \theta \in[-1,0), \\
\int_{-\tau}^{0} \eta(\theta, \mu) \varphi(\theta), & \theta=0,
\end{array}\right.
$$

and

$$
R(\mu) \varphi(\theta)= \begin{cases}0, & \theta \in[-1,0), \\ f(\mu, \theta), & \theta=0 .\end{cases}
$$

Apparently, system (3.8) is equivalent to

$$
\dot{u}(t)=A(\mu) u_{t}+R(\mu) u_{t},
$$

where $x_{t}(\theta)=x(t+\theta), \theta \in[-1,0]$, for $\psi \in C^{\prime}\left([-1,0],\left(R^{2}\right)^{*}\right)$. We further define the operator $A^{*}$ by

$$
A^{*}(\mu) \psi(s)=\left\{\begin{array}{lr}
-\frac{d \psi(s)}{d s}, & s \in(0,1] \\
\int_{-\tau}^{0} d \eta^{\top}(t, 0) \psi(-t), & s=0
\end{array}\right.
$$

and a bilinear form

$$
\langle\psi(s), \varphi(\theta)\rangle=\psi^{\top}(0) \varphi(0)-\int_{-1}^{0} \int_{\xi=0}^{\theta} \psi^{\top}(\xi-\theta) d \eta(\theta) \varphi(\xi) d \xi,
$$

where $\eta(\theta)=\eta(\theta, 0)$. Then $A^{*}(0)$ and $A(0)$ are adjoint operators. From the above analysis, we obtain that $\pm i \omega \tau_{k}$ are the eigenvalues of $A(0)$. Therefore they are also the eigenvalues of $A^{*}(0)$. Let $q(\theta)$ be an eigenvector of $A(0)$ corresponding to $i \omega \tau_{k}$, and $q^{*}(\theta)$ be an eigenvector of $A^{*}(0)$ corresponding to $-i \omega \tau_{k}$. Then we have $A(0) q(\theta)=i \omega \tau_{10} q(\theta)$ and $A^{*}(0) q^{*}(\theta)=-i \omega \tau_{10} q^{*}(\theta)$. Now suppose that $q(\theta)=\left(1, \rho_{1}, \rho_{2}\right)^{\top} e^{i \omega \tau_{k} \theta}$ is the eigenvectors of $A(0)$ corresponding to $i \omega \tau_{k}$. It follows from the definitions of $A(0), L_{\mu}(\varphi)$ and $\eta(0, \mu)$ that

$$
q(\theta)=\left(1, \rho_{1}, \rho_{2}\right)^{\top} e^{i \omega \tau_{k} \theta}=q(0) e^{i \omega \tau_{k} \theta},
$$

and similarly, by definition of $A^{*}$,

$$
q^{*}(\theta)=D\left(1, \gamma_{1}, \gamma_{2}\right)^{\top} e^{i \omega \tau_{k} \theta}=q^{*}(0) e^{i \omega \tau_{k} \theta} .
$$

To prove that $\left\langle q^{*}(s), q(\theta)\right\rangle=1$, we evaluate D. By the definition of bilinear inner product,

$$
\left\langle q^{*}(\theta), q(\theta)\right\rangle=\bar{D}\left(1, \bar{\gamma}_{1}, \bar{\gamma}_{2}\right)\left(1, \rho_{1}, \rho_{2}\right)^{\top}-\int_{-\tau}^{0} \int_{\xi=0}^{\theta} \bar{D}\left(1, \bar{\gamma}_{1}, \bar{\gamma}_{2}\right) e^{i \omega \tau_{k}(\xi-\theta)}
$$




$$
\begin{aligned}
& \times \mathrm{d} \eta(\theta)\left(1, \rho_{1}, \rho_{2}\right)^{\top} e^{i \omega \tau_{k} \xi} \mathrm{d} \xi \\
= & \overline{\mathrm{D}}\left\{1+\rho_{1} \bar{\gamma}_{1}+\rho_{2} \bar{\gamma}_{2}-\int_{-\tau}^{0}\left(1, \bar{\gamma}_{1}, \bar{\gamma}_{2}\right) \theta e^{i \omega \tau_{k} \theta} \mathrm{d} \eta(\theta)\right. \\
& \left.\times\left(1, \rho_{1}, \rho_{2}\right)^{\top}\right\} \\
= & \overline{\mathrm{D}}\left[1+\rho_{1} \bar{\gamma}_{1}+\rho_{2} \bar{\gamma}_{2}+\tau_{k} e^{i \omega \tau_{k}}\left(A_{2}+\mathrm{B}_{3} \rho_{2} \bar{\gamma}_{2}\right)\right] .
\end{aligned}
$$

Then we can choose $\overline{\mathrm{D}}$ as

$$
\overline{\mathrm{D}}=\left[1+\rho_{1} \bar{\gamma}_{1}+\rho_{2} \bar{\gamma}_{2}+\tau_{k} e^{i \omega \tau_{k}}\left(A_{2}+B_{3} \rho_{2} \bar{\gamma}_{2}\right)\right]^{-1},
$$

such that $\left\langle\mathrm{q}^{*}(\mathrm{~s}), \mathrm{q}(\theta)\right\rangle=1$ and $\left\langle\mathrm{q}^{*}(\mathrm{~s}), \overline{\mathrm{q}}(\theta)\right\rangle=0$.

In the remainder of this section, we use the same notation as in Hassard et al. [8] and compute the coordinates describing center manifold $\mathrm{C}_{0}$ at $\mu=0$.

Define

$$
z(t)=\left\langle q^{*}, u_{t}\right\rangle, \quad W(t, \theta)=u_{t}(\theta)-z q-\bar{z} \bar{q}=u_{t}(\theta)-2 \operatorname{Re}\{z(t) q(\theta)\}
$$

Regarding the center manifold $\mathrm{C}_{0}$, we have

$$
W(t, \theta)=W(z(t), \bar{z}(t), \theta)=W_{20}(\theta) \frac{z^{2}}{2}+W_{11}(\theta) z \bar{z}+W_{02}(\theta) \frac{z^{2}}{2}+\cdots,
$$

where $z$ and $\bar{z}$ are local coordinates for center manifold $C_{0}$ in the direction of $q$ and $\bar{q}^{*}$. If $u_{t}$ is real, we note that $W$ is real. We consider only real solutions. For the solution $u_{t} \in C_{0}$, since $\mu=0$ and (4.2), we can get

$$
\begin{aligned}
& \dot{z}=i \omega \tau z+\left\langle q^{*}(\theta), f(0, W(z, \bar{z}, \theta)+2 \operatorname{Re} z q(\theta))\right\rangle \\
& \stackrel{\text { def }}{=} i \omega \tau z+q^{*}(0) f_{0}(z, \bar{z})=i \omega \tau z+g(z, \bar{z}),
\end{aligned}
$$

where

$$
g(z, \bar{z})=q^{*}(0) f_{0}(z, \bar{z})=g_{20} \frac{z^{2}}{2}+g_{11} z \bar{z}+g_{02} \frac{\bar{z}^{2}}{2}+g_{21} \frac{z^{2} \bar{z}}{2}+\cdots
$$

By using (3.16), we have $x_{t}(\theta)=W(z, \bar{z}, \theta)+2 \operatorname{Re}\{z(t) q(\theta)\}$

$$
\begin{aligned}
x_{\mathrm{t}}=\left[\begin{array}{c}
x_{1 \mathrm{t}}(\theta) \\
x_{2 \mathrm{t}}(\theta) \\
x_{3 \mathrm{t}}(\theta)
\end{array}\right]= & {\left[\begin{array}{c}
W^{(1)}(z, \bar{z}, \theta) \\
W^{(2)}(z, \bar{z}, \theta) \\
W^{(3)}(z, \bar{z}, \theta)
\end{array}\right]+z\left[\begin{array}{c}
1 \\
\rho_{1} \\
\rho_{2}
\end{array}\right] e^{i \omega \theta}+\bar{z}\left[\begin{array}{c}
1 \\
\bar{\gamma}_{1} \\
\bar{\gamma}_{2}
\end{array}\right] e^{-i \omega \theta}, } \\
x_{1 \mathrm{t}}(\theta)= & z e^{i \omega \theta}+\bar{z} e^{-i \omega \theta}+W^{(1)} 20(\theta) \frac{z^{2}}{2} \\
+ & W^{(1)}{ }_{11}(\theta) z \bar{z}+W^{(1)} 02(\theta) \frac{z^{2}}{2}+\mathrm{O}\left(|z, \bar{z}|^{3}\right) \\
x_{2 \mathrm{t}}(\theta)= & z \rho_{1} e^{i \omega \theta}+\bar{z} \bar{\gamma}_{1} e^{-i \omega \theta}+W^{(2)} 20(\theta) \frac{z^{2}}{2} \\
& +W^{(2)} 11(\theta) z \bar{z}+W^{(2)} 02(\theta) \frac{z^{2}}{2}+O\left(|z, \bar{z}|^{3}\right) \\
x_{3 \mathrm{t}}(\theta)= & z \rho_{2} e^{i \omega \theta}+\bar{z} \bar{\gamma} 1 e^{-i \omega \theta}+W^{(2)} 20(\theta) \frac{z^{2}}{2} \\
& +W^{(2)} 11(\theta) z \bar{z}+W^{(2)} 02(\theta) \frac{z^{2}}{2}+O\left(|z, \bar{z}|^{3}\right)
\end{aligned}
$$


It follows that

$$
g(z, \bar{z})=\bar{q}^{*}(0) f_{0}(z, \bar{z})=\bar{D} \tau_{10}\left(1 \bar{\gamma}_{1} \bar{\gamma}_{2}\right)\left[\begin{array}{c}
f_{11} \\
f_{12} \\
f_{13}
\end{array}\right] .
$$

By comparing the coefficients with (4.10), we can get $g_{20}, g_{11}, g_{02}$ and $g_{21}$ and we need to compute $W_{20}(\theta)$ and $W_{11}(\theta)$ to determine $g_{21}$. By (4.1) and (4.10), we have

$$
\begin{array}{rlrl}
\dot{W} & =\dot{u}_{\mathrm{t}}-\dot{z} \mathrm{q}-\dot{\bar{z}} \bar{q} & \\
& = \begin{cases}A W-2 \operatorname{Re}\left(\bar{q}^{*}(\theta) f_{0} q(\theta)\right), & \theta \in[-1,0], \\
A W-2 \operatorname{Re}\left(\bar{q}^{*}(\theta) f_{0} q(\theta)\right)+f_{0}(z, \bar{z}), & \theta=0,\end{cases} \\
& =A W+H(z, \bar{z}, \theta), &
\end{array}
$$

where

$$
\mathrm{H}(z, \bar{z}, \theta)=\mathrm{H}_{20}(\theta) \frac{z^{2}}{2}+\mathrm{H}_{11}(\theta) z \bar{z}+\mathrm{H}_{02}(\theta) \frac{\bar{z}^{2}}{2}+\cdots .
$$

On the other hand, taking the derivative with respect to $t$ in (4.9), we can get

$$
\dot{W}=W_{z} \dot{z}+W_{\bar{z}} \dot{\bar{z}}
$$

Together with (4.13) and (4.14), this leads to

$$
\begin{aligned}
(A-2 i \omega \tau) W_{20}(\theta) & =-H_{20}(\theta), \\
A_{11}(\theta) & =-H_{11}(\theta), \\
\left(A+2 i \omega \tau_{10}\right) W_{02}(\theta) & =-H_{02}(\theta) .
\end{aligned}
$$

By using (4.8) for $\theta \in[-1,0]$, we can get

$$
\begin{aligned}
\mathrm{H}(z, \bar{z}, \theta) & =-\operatorname{Re} \bar{q}^{*}(\theta) \mathrm{f}_{0}(z, \bar{z}) \mathrm{q}(\theta) \\
& =-\mathrm{g}(z, \bar{z}) \mathrm{q}(\theta)-\bar{g}(z, \bar{z}) \overline{\mathbf{q}}(\theta) .
\end{aligned}
$$

Comparing the coefficient with (4.12), we can obtain

$$
H_{20}(\theta)=-g_{20} q(\theta)-\bar{g}_{02} \bar{q}(\theta), \quad H_{11}(\theta)=-g_{11} q(\theta)-\bar{g}_{11} \bar{q}(\theta) .
$$

From (4.14) and (4.16) and the definition of $A$, we get

$$
W_{20}(\theta)=\frac{i g_{20}}{\tau_{10} \omega} q(0) e^{i \omega \tau_{10} \theta}+\frac{i g_{02}}{3 i \omega} \bar{q}(0) e^{-i \omega \tau_{10} \theta}+E_{1} e^{\omega \theta} .
$$

Similarly, we have

$$
W_{11}(\theta)=\frac{i g_{11}}{\tau_{10} \omega} q(0) e^{i \omega \tau_{10} \theta}+\frac{i \bar{g}_{11}}{3 i \omega} \bar{q}(0) e^{-i \omega \tau_{10} \theta}+E_{2},
$$

where $E_{1}=\left(E_{1}^{(1)}, E_{1}^{(2)}\right) \in R^{2}, E_{2}=\left(E_{2}^{(1)}, E_{2}^{(2)}\right) \in R$ is a constant vector.

After finding $E_{1}$ and $E_{2}$, we can compute $W_{20}(\theta)$ and $W_{11}(\theta)$ from (4.19)-(4.20) and consider the following values to investigate the qualities of bifurcation periodic solution in the center manifold at the critical value $\tau_{k}$. To this end, we express each $g_{i j}^{\prime}$ in terms of the parameters and delay. Then we can calculate the following values:

$$
\left\{\begin{array}{l}
\mathrm{C}_{1}(0)=\frac{i}{2 \omega \tau}\left(g_{20} g_{11}-2\left|g_{11}\right|^{2}-\frac{\left|g_{02}\right|^{2}}{3}\right)+\frac{g_{21}}{2} \\
\mu_{2}=-\frac{\operatorname{Re}\left\{C_{1}(0)\right\}}{\operatorname{Re}\left\{\lambda^{\prime}(\tau)\right\}} \\
\beta_{2}=2 \operatorname{Re}\left\{C_{1}(0)\right\} \\
T_{2}=-\frac{\operatorname{Im}\left\{C_{1}(0)\right\}+\mu_{2} \operatorname{Im}\left\{\lambda^{\prime}(\tau)\right\}}{\omega} .
\end{array}\right.
$$

From above analysis, we can get the following theorem. 

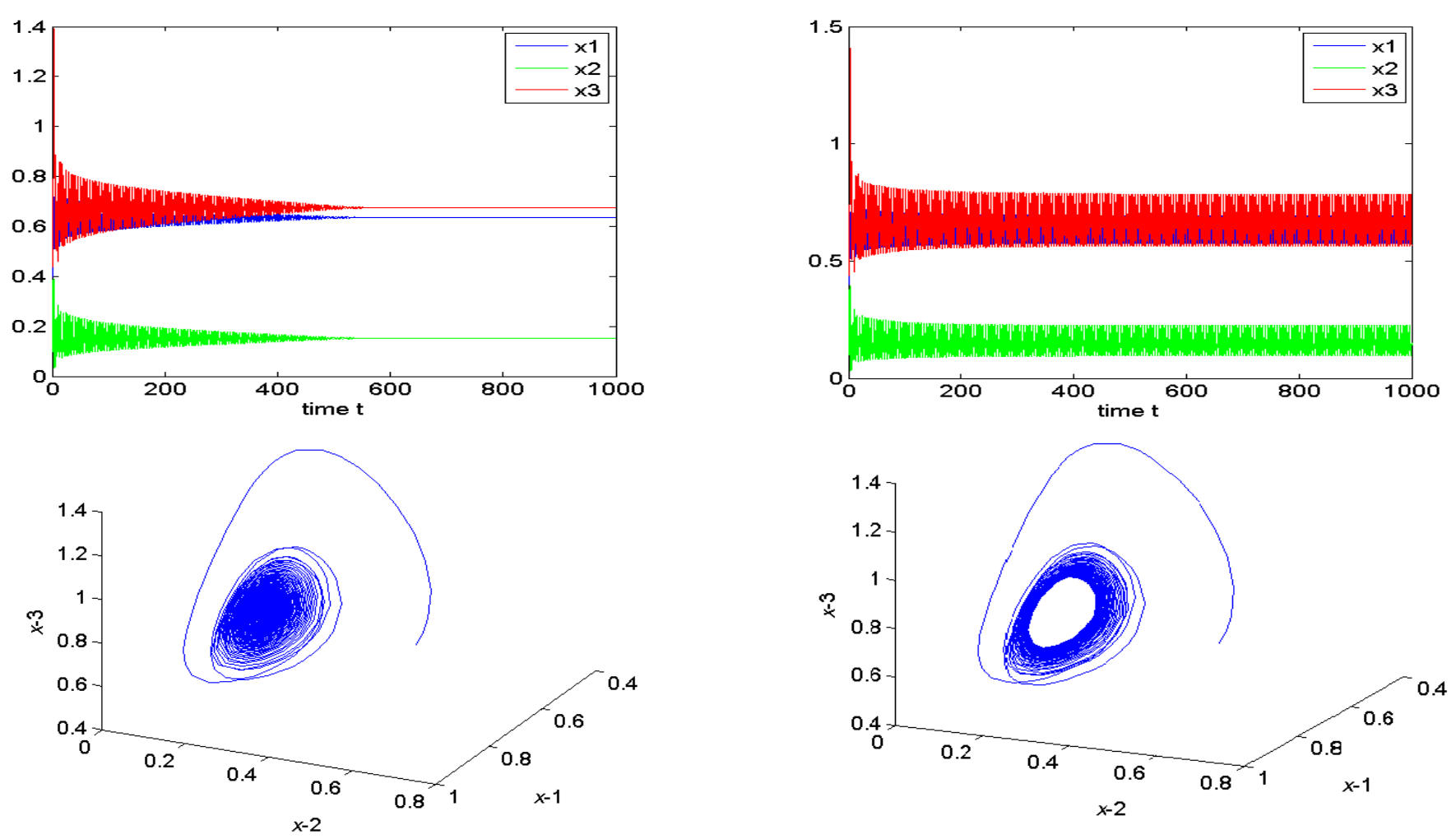

Figure 1: The positive equilibrium of system (1.2) is locally asymptotically stable for $\tau_{1}=\tau_{2}=\tau=2.3<2.31$ and the Hopf bifurcation occurs for $\tau_{1}=\tau_{2}=\tau=2.34>2.31$.

Theorem 4.1. When $\tau=\tau_{k}$, the stability and direction of periodic solution of the Hopf bifurcation of system (1.2) are determined by the parameters $\mu_{2}, \beta_{2}$ and $T_{2}$.

(i) The direction of the Hopf bifurcation is determined by the sign of $\mu_{2}$ : if $\mu_{2}>0\left(\mu_{2}<0\right)$, then the Hopf bifurcation is supercritical (subcritical), and a bifurcation periodic solution exists for $\tau>\tau_{0}\left(\tau<\tau_{0}\right)$.

(ii) The stability of the bifurcation periodic solution is determined by the sign of $\beta_{2}$ : if $\beta_{2}>0\left(\beta_{2}<0\right)$, then the bifurcation periodic solution is stable (unstable).

(iii) The sign of $T_{2}$ determines the period of the bifurcation periodic solution: if $T_{2}>0\left(T_{2}<0\right)$, then the period increases (decreases).

\section{Numerical simulations}

Next, we give some numerical examples to verify the above results. We consider system (1.2) with the following coefficients under different cases.

$$
\begin{aligned}
& \frac{d x}{d t}=x(1-x)-\frac{4 x}{1+0.1 x} y\left(t-\tau_{1}\right), \\
& \frac{d y}{d t}=-0.6 y+\frac{4 x}{1+0.1 x} y-\frac{4 x}{1+0.1 x} z\left(t-\tau_{2}\right), \\
& \frac{d z}{d t}=-0.7 z+\frac{4 x}{1+0.1 x} z .
\end{aligned}
$$

By simple calculation, we have $E^{*}=(1.2454,0.1523,0.9467)$. Firstly, we can get $\tau_{0}=2.31$ when $\tau_{1}=$ $\tau_{2}=\tau \neq 0$. According to Theorem 3.3, the corresponding waveforms and phase diagram are shown in Figure 1. Then, we have $\tau_{10}=2.58$ when $\tau_{2}=0$. And according to Theorem 3.6, we can get the corresponding waveforms and phase diagram, as shown in Figure 2. Next, we obtain $\tau_{20}=2.945$ when $\tau_{1}=0$. From Theorem 3.9, the corresponding waveforms and phase diagram are shown in Figure 3. Finally, by considering $\tau_{2}$ as a parameter and letting $\tau_{1}=2.5$ in its stable interval, we get that $E^{*}$ is locally asymptotically stable for $\tau_{2} \in\left(0, \tau_{30}\right)$ and unstable for $\tau_{2}>\tau_{30}$. And the waveforms and phase diagram are shown in Figure 4. 

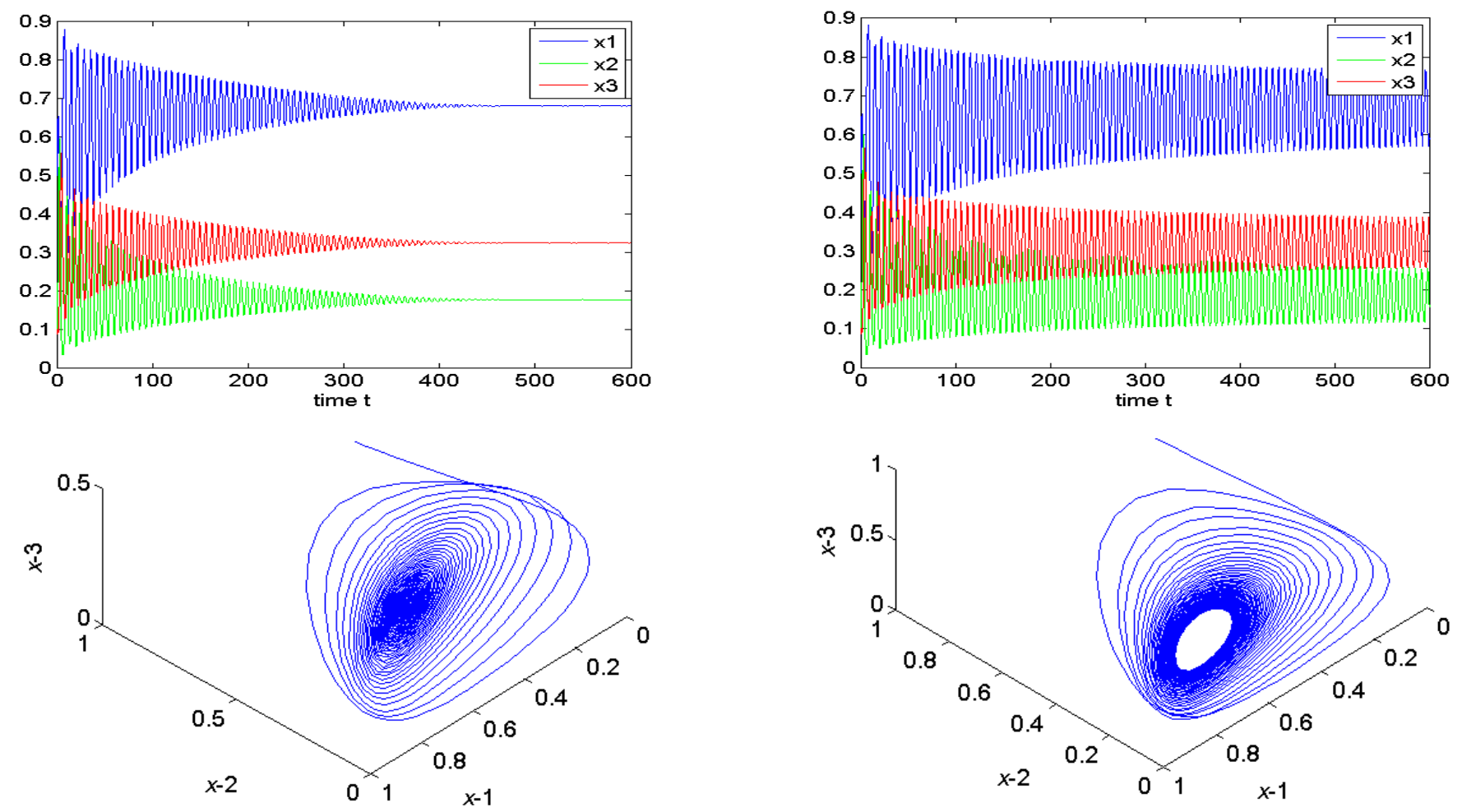

Figure 2: The positive equilibrium of system (1.2) is locally asymptotically stable for $\tau_{2}=0, \tau_{1}=2.5<2.58$ and the Hopf bifurcation occurs for $\tau_{2}=0, \tau_{1}=2.6>2.58$.
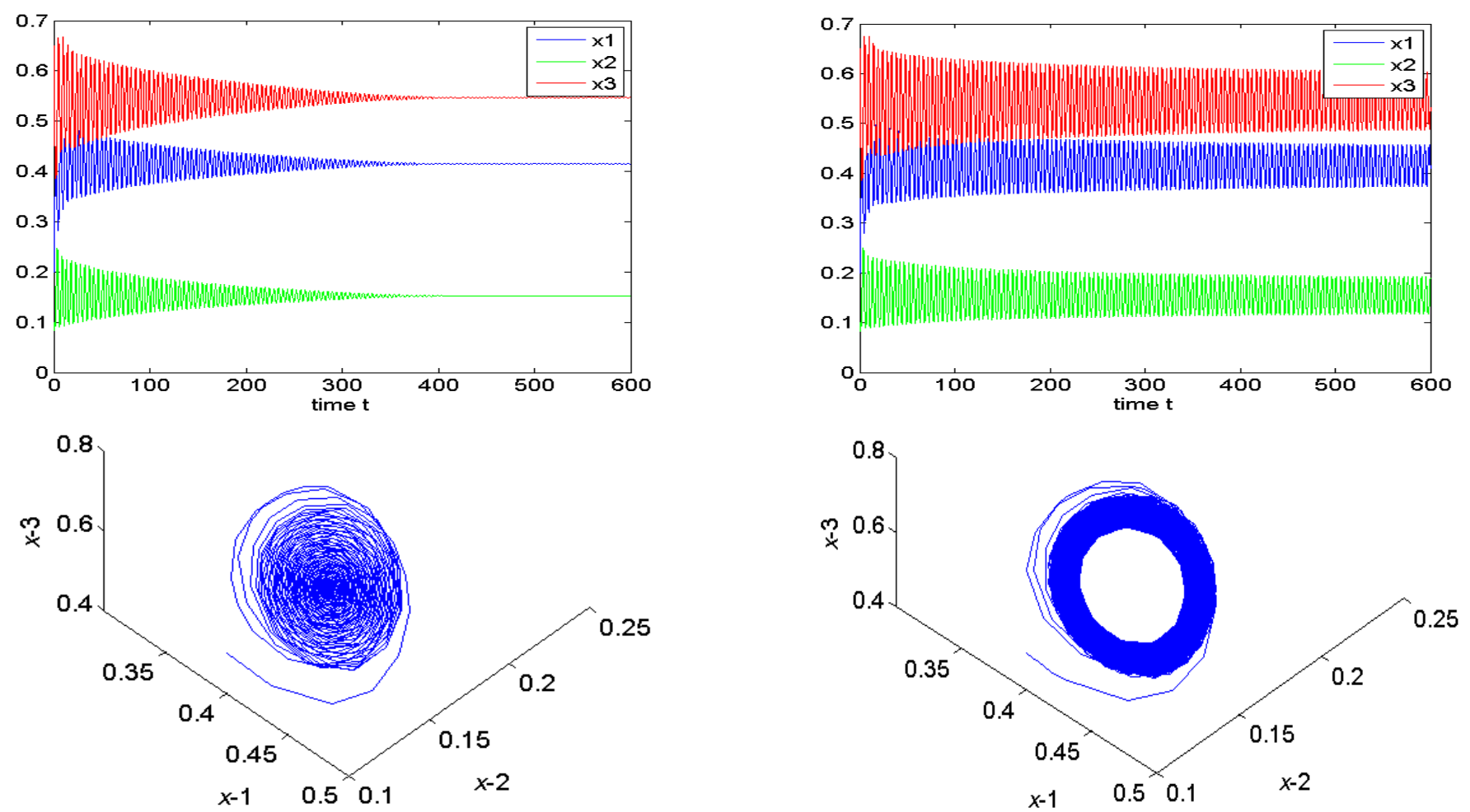

Figure 3: The positive equilibrium of system (1.2) is locally asymptotically stable for $\tau_{1}=0, \tau_{2}=2.9<2.945$ and the Hopf bifurcation occurs for $\tau_{1}=0, \tau_{2}=2.95>2.945$. 

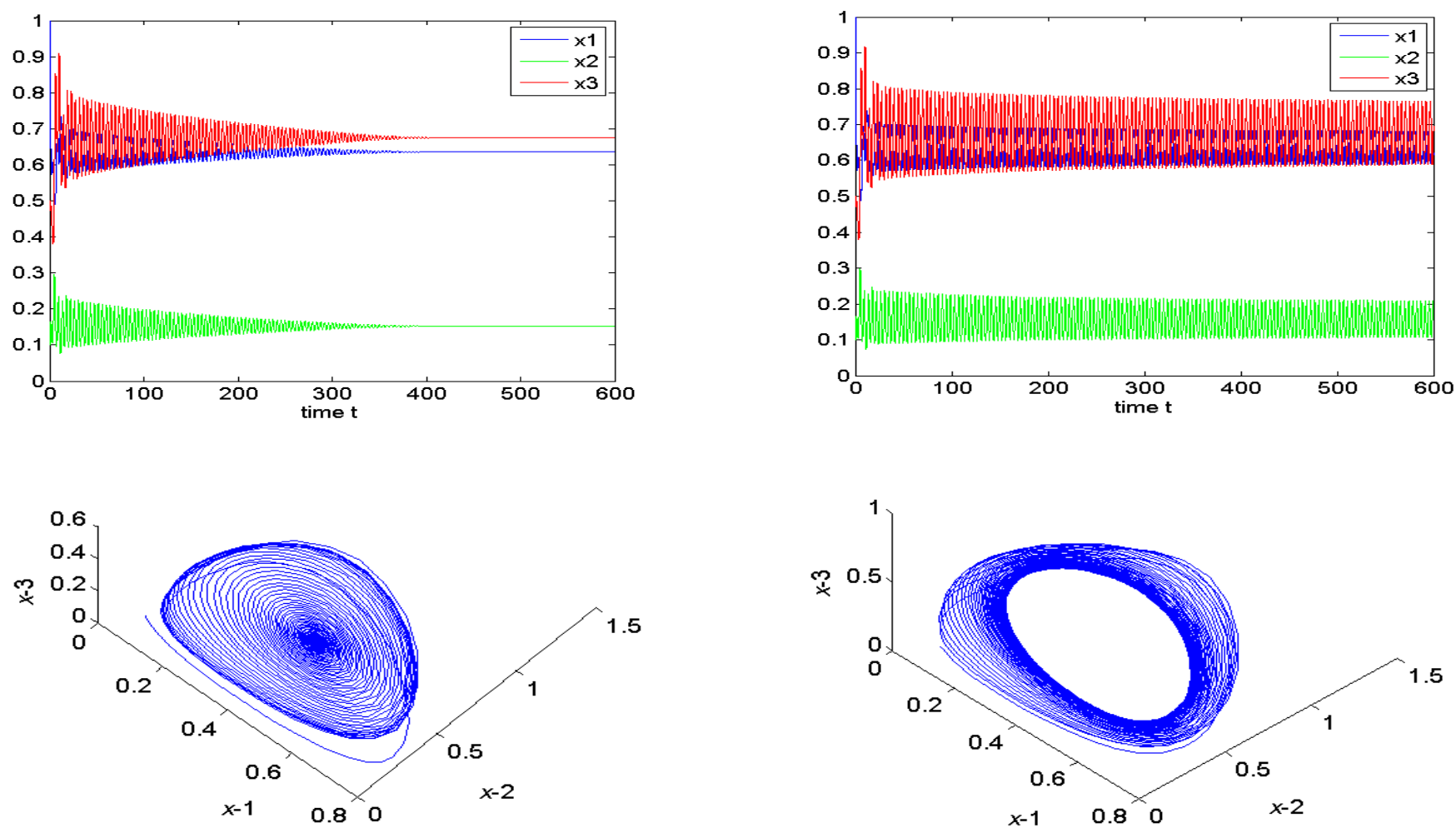

Figure 4: The positive equilibrium of system (1.2) is locally asymptotically stable for $\tau_{1}=2.5<2.58, \tau_{2}=2.35<2.37$ and the Hopf bifurcation occurs for $\tau_{1}=2.5<2.58, \tau_{2}=2.17>2.15$.

\section{Conclusions}

The existence of time delay would destroy the actual performance of the system and make the system unstable. The delay differential equations are a type of differential equation in which the derivative of the unknown function at a certain time is given in terms of the values of the function at previous times. The presence of time delay will make the dynamic behavior of the system more complex. Therefore, we provided a differential model to describe the dynamic of Hasting-Powell food chain system, and two different delays are incorporated into the model in the paper. The stability of equilibrium point and Hopf bifurcation are studied, and we also get the system's stability conditions. There are four cases in this paper that are discussed to illustrate the existence of Hopf bifurcation. Based on the center manifold theorem and the normal form theorem, we preliminary control the direction and stability of Hopf bifurcation. At last, we give numerical examples to verify the results. The existence of time delay is inevitable in real life and many nonlinear dynamic phenomena are affected by the time delay, so the study of nonlinear dynamic of time delay Hasting-Powell food chain model is particularly important.

\section{Acknowledgment}

The authors gratefully acknowledge the support from the National Natural Science Foundation (no. 613 64001), the Foundation of A Hundred Youth Talents Training Program of Lanzhou Jiaotong University, and the Specialized Research Fund for the Doctoral Program of Higher Education of China (no. 20136204110001).

\section{References}

[1] B. Bongiorno, N. Dinculeanu, The Riesz representation theorem and extension of vector valued additive measures, J. Math. Anal. Appl., 261 (2001), 706-732. 4 
[2] C. Califano, C. H. Moog, Accessibility of nonlinear time-delay systems, IEEE Trans. Automat. Control, 62 (2017), 1254-1268. 1

[3] C. Çelik, Stability and Hopf bifurcation in a delayed ratio dependent Holling-Tanner type model, Appl. Math. Comput., 255 (2015), 228-237. 1

[4] Y.-Y. Chen, J. Yu, C.-J. Sun, Stability and Hopf bifurcation analysis in a three-level food chain system with delay, Chaos Solitons Fractals, 31 (2007), 683-694. 1

[5] K. P. Das, S. Chatterjee, J. Chattopadhyay, Disease in prey population and body size of intermediate predator reduce the prevalence of chaos-conclusion drawn from Hastings-Powell model, Ecol. Complexity, 6 (2009), 363-374. 1

[6] S. Gakkhar, A. Singh, Control of chaos due to additional predator in the Hastings-Powell food chain model, J. Math. Anal. Appl., 385 (2012), 423-438. 1

[7] J. K. Hale, Functional differential equations, Applied Mathematical Sciences, Springer-Verlag, New York-Heidelberg, (1971). 3

[8] B. D. Hassard, N. D. Kazarinoff, Y. H. Wan, Theory and Applications of Hopf Bifurcation, London Math. Soc. Lect. Note Ser., Cambridge University Press, Cambridge-New York, (1981). 4

[9] A. Hastings, T. Powell, Chaos in a threespecies food chain, Ecology, 72 (1991), 896-903. 1

[10] A. Huseynov, The Riesz representation theorem on time scales, Math. Comput. Modelling, 55 (2012), 1570-1579. 4

[11] Z.-J. Li, Z.-T. Chen, J. Fu, C.-Y. Sun, Direct adaptive controller for uncertain MIMO dynamic systems with time-varying delay and dead-zone inputs, Automatica J. IFAC, 63 (2016), 287-291. 1

[12] A. E. Matouk, A. A. Elsadany, E. Ahmed, H. N. Agiza, Dynamical behavior of fractional-order Hastings-Powell food chain model and its discretization, Commun. Nonlinear Sci. Numer. Simul., 27 (2015), 153-167. 1

[13] C.-R. Tian, L. Zhang, Hopf bifurcation analysis in a diffusive food-chain model with time delay, Comput. Math. Appl., 66 (2013), 2139-2153. 1

[14] M. C. Varriale, A. A. Gomes, A study of a three species food chain, Ecol. Model., 110 (1998), 119-133. 3

[15] Z.-X. Yu, Y. Dong, S.-G. Li, F.-F. Li, Adaptive tracking control for switched strict-feedback nonlinear systems with timevarying delays and asymmetric saturation actuators, Neurocomputing, 238 (2017), 245-254. 1

[16] L.-Y. Zhang, Hopf bifurcation analysis in a Monod-Haldane predator-prey model with delays and diffusion, Appl. Math. Model., 39 (2015), 1369-1382. 1

[17] Y.-M. Zhang, J.-D. Cao, W.-Y. Xu, Stability and Hopf bifurcation of a Goodwin model with four different delays, Neurocomputing, 165 (2015), 144-151. 1

[18] Y.-J. Zhang, Y.-S. Ou, X.-Y. Wu, Y.-M. Zhou, Resilient dissipative dynamic output feedback control for uncertain Markov jump Lur'e systems with time-varying delays, Nonlinear Anal. Hybrid Syst., 24 (2017), 13-27.

[19] Z.-X. Zhong, J.-Y. Yu, Y.-D. He, T. Hayat, F. Alsaadi, Fuzzy-model-based decentralized dynamic-output-feedback $\mathrm{H}^{\infty}$ control for large-scale nonlinear systems with time-varying delays, Neurocomputing, 173 (2016), 1054-1065. 1 